\title{
Some Quantum Mechanical Relations in case of Singular Operators
}

\section{Teimuraz Nadareishvili ${ }^{1}$}

Faculty of Exact and Natural Sciences, Iv.Javakhishvili Tbilisi State University

I.Cavchavadze Ave 3, Tbilisi, Ceorgia,0179

E-mail: teimuraz.nadareishvili@tsu.ge

\section{Anzor Khelashvili}

Inst. of High Energy Physics, Iv. Javakhishvili Tbilisi State University

G.Danelia str 10, Tbilisi, Georgia;0186

E-mail: anzor.khelashvilietsu.ge

\begin{abstract}
Elaboration of some fundamental relations in 3-dimensional quantum mechanics is considered taking into account the restricted character of areas in radial distance. In such cases the boundary behavior of the radial wave function and singularity of operators at the origin of coordinates contribute to these relations. We derive the relation between the average value of the operator's time derivative and the time derivative of this operator, which is usually considered to be the same by definition. The deviation from the known result is deduced and manifested by extra term, which depends on the boundary behaviour mentioned above. The general form for this extra term takes place in the hypervirial-like theorems. As a particular case, the the virial theorem for Coulomb and oscillator potentials is considered and correction to the Kramers' sum rule is derived. Moreover the corrected Ehrenfest theorem is deduced and its consistency with real physical picture is demonstrated.
\end{abstract}

European Physical Society Conference on High Energy Physics - EPS-HEP2019 -

10-17 July, 2019

Ghent, Belgium

\footnotetext{
${ }^{1}$ Speaker

(C) Copyright owned by the author(s) under the terms of the Creative Commons

Attribution-NonCommercial-NoDerivatives 4.0 International License (CC BY-NC-ND 4.0).
} 


\section{Introduction}

In textbooks of quantum mechanics the most formulations are concerned mainly to the one dimensional problems and in these cases, as a rule, the wave functions decrease at infinity. It is clear when the system is located in finite volume, the inclusion of boundary conditions becomes necessary. The aim of this article is to study some quantum mechanical theorems in spherical coordinates, when the area is not a full space. We will see that in most cases problems arises when the potential in the Schrodinger equation is singular or the operators are singular itself.

\section{Time derivative of mean values of operators}

In quantum mechanics derivative of time-dependent operator is [1]

$$
\frac{d \hat{A}}{d t}=\frac{\partial \hat{A}}{\partial t}+\frac{i}{\hbar}[\hat{H}, \hat{A}]
$$

If one averages (2.1) by the state function, it follows

$$
\left\langle\frac{d \hat{A}}{d t}\right\rangle=\left\langle\frac{\partial \hat{A}}{\partial t}\right\rangle+\frac{i}{\hbar}\langle[\hat{H}, \hat{A}]\rangle
$$

As a rule one believes that these two operations - time derivative and average procedures can be interchanged. This is postulated as a definition [1]

$$
\left\langle\frac{d \hat{A}}{d t}\right\rangle=\frac{d\langle\hat{A}\rangle}{d t}=\left\langle\frac{\partial \hat{A}}{\partial t}\right\rangle+\frac{i}{\hbar}\langle[\hat{H}, \hat{A}]\rangle
$$

We show, that is not it true in general, in 3-dimensional space. Indeed calculate the derivate

$$
\frac{d\langle A\rangle}{d t}=\frac{d}{d t}\langle\psi|\hat{A}| \psi\rangle=\left\langle\frac{\partial \psi}{\partial t}|\hat{A}| \psi\right\rangle+\left\langle\psi\left|\frac{\partial \hat{A}}{\partial t}\right| \psi\right\rangle+\left\langle\psi|\hat{A}| \frac{\partial \psi}{\partial t}\right\rangle
$$

We'll use the time dependent Schrodinger eqation and its complex conjugate in the first and third terms of eq. (2.4), take the Hamiltonian in the radial form and performing twofold partial integration in the derivative terms of integrand, we obtain

$$
\frac{d\langle\hat{A}\rangle}{d t}=\frac{i}{\hbar}\langle[\hat{H}, \hat{A}]\rangle+\left\langle\frac{\partial \hat{A}}{\partial t}\right\rangle+\Pi
$$

where we have obtained the additional term

$$
\Pi=i \frac{\hbar}{2 m} \lim _{r \rightarrow 0}\left\{r^{2}\left[\hat{A} R \frac{d R^{*}}{d r}-R^{*} \frac{d}{d r}(\hat{A} R)\right]\right\}
$$

\section{Analysis of the additional term}

It is known that under general requirements the radial function must behave like

$$
\lim _{r \rightarrow 0} r R(r)=0
$$


This condition is the Dirichlet condition for reduced $u=r R$ wave function [3-4]. The following classification of potentials is well known [4-5]:

(1). Regular potentials:

$$
\lim _{r \rightarrow 0} r^{2} V=0
$$

For which solution at the origin behaves like

$$
\lim _{r \rightarrow 0} R=C_{1} r^{l}+C_{2} r^{-(l+1)}
$$

Clearly only first term satisfy (3.1), therefore it must be retained

(2).Singular potentials:

$$
\lim _{r \rightarrow 0} r^{2} V(r)= \pm \infty
$$

For them the "falling to the center" happens and is not interesting for us now.

(3) "Soft" singular potentials:

$$
\lim _{r \rightarrow 0} r^{2} V(r)= \pm V_{0} ;\left(V_{0}=\text { const }>0\right)
$$

For such potential the wave function has the following behavior [3-4]:

$$
\lim _{r \rightarrow 0} R=a_{s t} r^{-1 / 2+P}+a_{\text {add }} r^{-1 / 2-P}=R_{s t}+R_{\text {add }} ; P=\sqrt{(l+1 / 2)^{2}-2 m V_{0} / \hbar^{2}}
$$

For $0<\mathrm{P}<1 / 2$ the second solution satisfies the condition (3.1), therefore it must be retained and hence the self adjoint extension need to be performed [4].For $P>1 / 2$ the first solution remains. It is clear from Eq. (2.6) that the singularity of the operator at the origin will be important. We take it as

$$
\hat{A}(r) \sim 1 / r^{\beta} ; \beta>0
$$

Then, we have for regular potentials

$$
\Pi_{s t}=\frac{i \hbar C_{1}^{2}}{2 m} \lim _{r \rightarrow 0} r^{2 l+1-\beta}
$$

For $21+1>\beta$ the additional term vanishes. In opposite case the divergent result will follow and we will be unable to write the equation (2.2). For $21+1=\beta$ the extra term survives and we get

$$
\frac{d\langle A\rangle}{d t}=\left\langle\frac{\partial \hat{A}}{\partial t}\right\rangle+\frac{i}{\hbar}\langle[\hat{H}, \hat{A}]\rangle+\frac{i \hbar C_{1}}{m}\left(l+\frac{1}{2}\right)
$$

For potential (3.5) and regular solution $\mathrm{R}=\mathrm{R}_{\mathrm{st}}=a_{s t} r^{-1 / 2+P}$ we obtain

$$
\Pi_{s t}=i \hbar \frac{a_{s t}^{2} \beta}{2 m} \lim _{r \rightarrow 0} r^{2 P-\beta}
$$

Here also we get $\Pi_{s t}=0$, when $2 \mathrm{P}>\beta$. But for $2 \mathrm{P}=\beta$ the finite contribution follows 


$$
\frac{d\langle\hat{A}\rangle}{d t}=\left\langle\frac{\partial \hat{A}}{\partial t}\right\rangle+\frac{i}{\hbar}\langle[\hat{H}, \hat{A}]\rangle+\frac{i \hbar a_{s t}^{2}}{m} P
$$

Conclusion: This "strange" result is provided by singular character of the operator. The strangest is the fact that the time derivative of average value does not coincide to the average of derivative of the operator

$$
\left\langle\frac{d \hat{A}}{d t}\right\rangle=\frac{d\langle\hat{A}\rangle}{d t}-\Pi
$$

Moreover, many famous theorems like Ehrenfest or hypervirial relations may be modified.[6-7]

\section{Stationary states and integrals of motion}

For stationary states wave function has the following dependence

$$
\psi(\vec{r}, t)=e^{-\frac{i}{\hbar} E t} \phi(\vec{r})
$$

When the operator also doesn't explicitly dependent on time, we have an operator equality

$$
\frac{d \hat{A}}{d t}=\frac{i}{\hbar}[\hat{H}, \hat{A}]
$$

Averaging this equality by means of (4.1), we get

$$
\left\langle\frac{d \hat{A}}{d t}\right\rangle=\frac{i}{\hbar}\left\{\int_{0}^{\infty} R^{*} \hat{H} \hat{A} R r^{2} d r-E \int_{0}^{\infty} R^{*} \hat{A} R r^{2} d r\right\}
$$

Let us consider two cases : (a). $\hat{A}$ commutes with $\hat{\mathrm{H}}$. Then it follows from (4.3), that

$$
\left\langle\frac{d \hat{A}}{d t}\right\rangle=\frac{d\langle\hat{A}\rangle}{d t}=0
$$

So in this case, the mean value of this operator is conserved and is an integral of motion.

(b) When Hamiltonian does not commute with the operator, we derive

$$
\langle d \hat{A} / d t\rangle=-\Pi
$$

On the other hand, when operator is independent of time explicitly, we have

$$
\langle A\rangle=\int \phi^{*}(\vec{r}) \hat{A} \phi(\vec{r}) d \vec{r}
$$

Evidently

$$
\langle d \hat{A} / d t\rangle \neq 0
$$

So we obtain "strange" result : for stationary states, when $\hat{A} \hat{H} \neq \hat{H} \hat{A}$, Eq. (4.7) is valid or $\hat{A}$ is conserved, but according to Eq. (4.5), $\langle\mathrm{d} \hat{\mathrm{A}} / \mathrm{dt} \neq 0\rangle$. Therefore, we conclude that the definition $\overline{\dot{\mathrm{f}}}=\dot{\overline{\mathrm{f}}}$, given initially, depends on the singularity of the considered operator. Remark, that this point (operator's singularity) was not discussed in the literature up to now. 


\section{Modified hypervirial theorems}

Comparing Eqs. (4.3) and (4.9), one derives

$$
\frac{i}{\hbar}\langle[\hat{H}, \hat{A}]\rangle=-\Pi
$$

The usual hypervirial theorem is formulated as [7-9]: If $\phi$ is a bound state eigenfunction of the $\hat{H}$ and if $\hat{A}$ is an arbitrary Hermitian time - independent operator, then $\langle\phi,[\hat{\mathrm{H}}, \hat{\mathrm{A}}] \phi\rangle=0$ This theorem must be modified and according to Eq. (5.1) it have the following form:

$$
\langle\phi,[\hat{H}, \hat{A}] \phi\rangle=i \hbar \Pi
$$

Here the choice of the operator is very important. Consider the following operator [8]

$$
\hat{A}=\hat{p}_{r} r^{S+1}
$$

where $\hat{\mathrm{p}}_{\mathrm{r}}$ is a radial momentum(hermitian) operator [2]

We obtain for (3.6) potentials

$\left.\left.4 m / \hbar^{2}\left\{\left\langle r^{s+1} d V / d r\right\rangle+2(s+1) \mid\left\langle r^{s} V\right\rangle-E\left\langle r^{s}\right\rangle\right\}\right\}+s \mid(2 l+1)^{2}-s^{2}\right\}\left\langle r^{s-2}\right\rangle=a_{s t}^{2} s(s-2 P) \delta_{2 P,-s}$

For the regular potential (3.2) we have

$\left.2 m / \hbar^{2}\left\{\left\langle r^{s+1} d V / d r\right\rangle+2(s+1)\left[\left\langle r^{s} V\right\rangle-E\left\langle r^{s}\right\rangle\right\}\right\}+1 / 2 s \mid(2 l+1)^{2}-s^{2}\right\}\left\langle r^{s-2}\right\rangle=(2 l+1)^{2} C_{l}^{2} \delta_{s+1,-2 l}$

\section{Modification of the Ehrenfest theorem}

We now analyze what happens with the Ehrenfest theorem in light of the influence of presented boundary behavior .Substitute the radial momentum operator into Eq.(2.5), we have

$$
d\left\langle\hat{p}_{r}\right\rangle / d t=i / \hbar\left\langle\left[\hat{H}, \hat{p}_{r}\right]\right\rangle+\Pi_{s t}
$$

where

$$
\Pi_{s t}=a_{s t}^{2} \hbar^{2} / 2 m(1 / 2+P) \lim _{r \rightarrow 0} r^{2 P-1}
$$

For $2 \mathrm{P}>1, \Pi_{\mathrm{st}}=0$, while for $2 \mathrm{P}<1$, it diverges. For $2 \mathrm{P}=1$ it survives $\Pi_{\mathrm{st}}=a_{s t}^{2} \hbar^{2} / 2 \mathrm{~m}$.

So the usual Ehrenfest theorem works only for $2 P>1$.In textbooks this fact isn't mentioned.

Let us now calculate the commutator in (6.1). We find

$$
\left\lfloor\hat{H}, \hat{p}_{r}\right\rfloor=-i \hbar^{3} l(l+1) / m r^{3}-i \hbar F_{r}
$$

Where $\mathrm{F}_{\mathrm{r}}$ is a "radial force". Therefore we obtain the modified Ehrenfest theorem

$$
\frac{d\left\langle p_{r}\right\rangle}{d t}=\frac{\hbar^{2} l(l+1)}{m}\left\langle\frac{1}{r^{3}}\right\rangle+\left\langle F_{r}\right\rangle+\Pi_{s t}
$$

In stationary case the left-hand side of (6.4) must be zero. So we should have $C_{1}^{2} \hbar^{2} / 2 m=2 e^{2} / n^{3} a_{0}^{2}$ and it follows a correct expression for Bohr's first orbit radius and $a_{0}=\hbar^{2} / m e^{2}$. We conclude that in Eq. (6.4) the term $\Pi_{\text {st }}$ is necessary for deriving correct, results, which is absent in [10-11]. Lastly, it is easy to show, that for the $\hat{\mathrm{A}}=\hat{\mathrm{r}}, \Pi_{\mathrm{st}}=0$ and the Ehrenfest theorem has usual $\mathrm{d}\langle\mathrm{r}\rangle / d t=\left\langle p_{r}\right\rangle / m$ form. The obtained results are easily understand able, because the $\hat{\mathrm{p}}_{\mathrm{r}}$ is singular at the origin in spite of the coordinate operator. 


\section{Conclusions}

1) We considered an influence of the restricted region in 3-dimensional space. We show that boundary behavior of radial function and singularity of operators contributes to the several fundamental relations. 2) We calculate this extra term and investigate, when it changes fundamental relations. 3) We show that the inclusion of the extra term is necessary to avoid some misunderstandings.

This work was supported by Shota Rustaveli National Science Foundation (SRNSF) [grant number № DI-2016-26, Project Title: ”Three-particle problem in a box and in the continuum"].

\section{References}

[1] L. Landau, E.Lifshitz, Quantum Mechanics, Pergamon, Oxford 1977.

[2] A.Messiah, Quantum Mechanics, Dover Publications, New York 1999

[3] A.Khelashvili A, T.Nadareishvili, What is the Boundary Condition for the Radial Wave Function of the Schrodinger Equation?, Am. J. Phys 79 (2011) 668. B

[4] A.Khelashvili,T. Nadareishvili, Singular Behavior of the Laplace Operator in Polar Spherical Coordinates and Some of Its Consequences for the Radial Wave Function at the Origin of Coordinates, Phys. of Particles and Nuclear Lett 12 (2015) 11.

[5] R. Newton, Scattering Theory of waves and Particles. Dover Publications, New York, 2002.

[6] Z.Ehrenfest, Bemerkung über die angenäherte Gültigkeit der klassischen Mechanik innerhalb der Quantenmechanik, Z.Phys 45 (1927) 455.

[7] O.Hirschfelder, Classical and quantum mechanical hypervirial theorems, J.Chem Physics. 33 (1960)1462

[8] S.Epstein, O.Hirschfelder, Hypervirial theorems for variational wave functions, Phys.Rev. 123 (1961) 1495.

[9] O.Hirschfelder, C.Coulson. Hypervirial theorems applied to molecular quantum mechanics, J.Chem.Physics 36 (1962) 941.

[10] U.Roy,S.Ghosh, T.Shreecharan,$\underline{\mathrm{K} . \text { Bhattacharya, Reality of linear and angular }}$ momentum expectation values in bound states [quant-ph/0704.0373].

[11] U.Roy, S. Ghosh, K. Bhattacharya, Some intricacies of the momentum operator in quantum mechanics [quant-ph/0706.0924] 